\title{
Route Planning Based on Genetic Algorithm
}

\author{
Lin $\operatorname{Li}^{1} \&$ Yuhua Zhang ${ }^{2}$ \\ ${ }^{1}$ School of TranslationStudies, Jinan University, Zhuhai, China \\ ${ }^{2}$ School of Electric\&Information, Jinan University, Zhuhai, China \\ Correspondence: Lin Li, School of TranslationStudies, Jinan University, Zhuhai, China.
}

Received: January 26, 2018 Accepted: February 9, 2018 Online Published: March 13, 2018

doi:10.5539/jmr.v10n2p122ＵRL: https://doi.org/10.5539/jmr.v10n2p122

\begin{abstract}
This paper mainly deals with the planning of aviation route and needs to determine the model to find out the shortest path. In this paper, we combine the methods of simulated annealing and genetic algorithm, and obtained the optimal solution method. Firstly, Genetic Algorithm (GA) uses the modified circle algorithm to find some feasible solutions to the approximate initial population, and then transforms them through simulated and crossover operations. This paper also introduces the aircraft fuel consumption model and the cubical smoothing algorithm with five-point approximation to reduce the aircraft fuel consumption and parts loss. The simulation results show that the accuracy of the route planning based on genetic algorithm is higher, while consumes less fuel and takes less sharp turns.
\end{abstract}

Keywords: route planning,modified circle algorithm,genetic algorithm

\section{Introduction}

Route planning is to find the optimal path for moving objects from the starting point to the target point under certain constraints, satisfying certain performance indexes and certain constraints. UAV route planning is one of the key technologies of virtual simulation training system. It mainly looks for the route from the starting point to the destination of tasks, under the constraints of scheduled tasks, threat distribution, fuel economy, etc. It plans in a dynamic, uncertain and real-time environment, which based on the terrain and enemy information. The current global route planning methods include particle swarm optimization, VORONOI, A * algorithm, ant colony algorithm, genetic algorithm and so on. (Wei Shui, 2011:574-576) In this paper, the problem of route planning based on genetic algorithm is proposed to simulate the requirements of the actual battlefield environment. The superiority of genetic algorithm in applying such problems is demonstrated.

\section{Method}

Genetic Algorithm is a computational model of biological evolution that simulates natural selection and genetics of Darwin's biological evolution. It is a method of searching for the optimal solution by simulating natural evolution. It was first proposed by Professor J. Holland in the United States in 1975. And it has been widely used in fields such as combinatorial optimization, machine learning, signal processing, adaptive control and artificial life. It is the key technology in modern intelligent computing. The route planning without constraint is essentially a question of a weighted undirected graph. It is required to find a loop that passes all the target points and finally returns to the starting point, so as to make each weight of the loop as small as possible. It is similar to the traveling salesman problem(Shuixi Wang, 2014), (Lingyi Kong, 2013). We can use annealing, genetic computing to analyze it.

\subsection{Shortest Route Modeling}

In order to simplify the problem, assuming that the flight route of a plane is on a horizontal two-dimensional plane without taking into account the flight altitude. Then a plane rectangular coordinate system is established, and the coordinates of our military airport in this coordinate system are $(1,1)$. The airplane is a particle, regardless of aircraft shape and size. The aircraft is flying at a constant speed, irrespective of the turning radius of the aircraft. Considering the distance between every two points as a population, the problem of choosing the smallest route can be understood as choosing the smallest population of $\mathrm{M}$ individuals in the population to evolve to the next generation, thus preserving the better characteristics. The parameters of the genetic algorithm are set as follows: Population $\mathrm{M}=50$; maximum algebra $\mathrm{G}=1000$; The crossover $P_{c}=1$ rate is 1 , which can ensure the full evolution of the population. The $P_{m}=0.1$ rate of mutation. In general, mutation is less likely to occur. 


\subsection{Coding Strategy}

We adopt decimal-coded, using random number sequence $\omega_{1} \omega_{2} \cdots \omega_{14}$ as chromosomes. In which, $0 \leq \omega_{i} \leq 1(i=$ $2,3, \ldots, 14), \omega_{1}=0, \omega_{14}=1$; each random sequence corresponds to an individual in the population.

\subsection{Route Planning Based on Genetic Algorithm}

With a known range, a known target point and starting point, the steps of planning the shortest route of the aircraft is as follows:

\subsubsection{The Initial Population}

First, use the classical approximation algorithm - modified circle algorithm to find a better initial population: Find the initial circle Find out the point that has shortest distance to the airport point among all the target points. Then use the found target point as a starting point and then find out the point has nearest distance from it. Repeat this until all the target points have been covered, and finally come back to the airport point. The path obtained in this way is the initial circle. Find the improvement circle Using the initial circle obtained as a Hamilton cycle $\mathrm{C}$ of the modified circle algorithm, and then modify $\mathrm{C}$ to obtain another Hamilton circle with a smaller weight. Set the initial circle

$$
C=v_{1} v_{2} \cdots v_{i} v_{i+1} \cdots v_{j} v_{j+1} \cdots v_{n} v_{1}
$$

For $1 \leq i<i+1<j<j+1<n$, construct a new Hamilton circle

$$
C_{i j}=v_{1} v_{2} \cdots v_{i} v_{j} v_{j-1} v_{j-2} \cdots v_{i+1} v_{j+1} v_{j+2} \cdots v_{n} v_{1}
$$

It is obtained by removing the edge $v_{i} v_{i+1}$ and $v_{j} v_{j+1}$ from $\mathrm{C}$, and adding edge $v_{i} v_{j}$ and $v_{i+1} v_{j+1}$. If

$$
\omega\left(v_{i} v_{j}\right)+\omega\left(v_{i+1} v_{j+1}\right)<\omega\left(v_{i} v_{i+1}\right)+\omega\left(v_{j} v_{j+1}\right)
$$

We replace $\mathrm{C}$ by $C_{i j}, C_{i j}$ was called C's improvement circle. Continue to repeat, until it can not be improved. For the randomly generated initial circle

$$
C=\pi_{1} \cdots \pi_{u-1} \pi_{u} \pi_{u+1} \cdots \pi_{v-1} \pi_{v} \pi_{v+1} \cdots \pi_{14}
$$

Thereinto, $2 \leq u<v \leq 13,2 \leq \pi_{u}<\pi_{v} \leq 13$. Exchange the order between $\mathrm{u}$ and $\mathrm{v}$. As a result, the new path is

$$
\pi_{1} \cdots \pi_{u-1} \pi_{v} \pi_{v-1} \cdots \pi_{u+1} \pi_{u} \pi_{v+1} \cdots \pi_{14}
$$

Regard it as $\Delta f=\left(d_{\pi_{u-1} \pi_{v}}+d_{\pi_{u} \pi_{v+1}}\right)-\left(d_{\pi_{u-1} \pi_{u}}+d_{\pi_{v} \pi_{v+1}}\right)$, If $\Delta f<0$, then turn the old path into a new path, until it can not be modified so far. As a result, we get a feasible solution. Until $\mathrm{M}$ feasible solutions are generated, the $\mathrm{M}$ feasible solutions are converted into chromosome codes.

\subsubsection{The Objective Function}

The objective function is to provide the length of route while detecting all the targets point, and the fitness function is taken as the objective function. We require

$$
\min f\left(\pi_{1}, \pi_{2}, \cdots, \pi_{14}\right)=\sum_{i=1}^{13} d_{\pi_{i} \pi_{i+1}}
$$

\subsubsection{Crossover Operation}

We use a single point crossover in crossover operation. For two selected parent individuals $f_{1}=\omega_{1} \omega_{2} \cdots \omega_{14}$ and $f_{2}=\omega_{1}{ }^{\prime} \omega_{2}{ }^{\prime} \cdots \omega_{14}{ }^{\prime}$, the random selection of the $\mathrm{t}$-th gene is the crossover point, then the child individuals obtained by crossover operation after are $s_{1}$ and $s_{2}$. The gene of $s_{1}$ consists of the first $\mathrm{t}$ genes of $f_{1}$ and the last 14-t gene of $f_{2}$. The gene of $s_{2}$ consists of the first t genes of $f_{2}$ and the last 14-t genes of $f_{1}$. There are many ways to crossover, we choose the best way to ensure that offspring can inherit the excellent characteristics of their parents. At the same time, the crossover operation also contains mutation operation.

\subsubsection{Mutation Operation}

Mutation is also a means of achieving diversity in the community and a guarantee of optimization. According to the given mutation rate, Randomly take three integers among the selected mutation individuals, which satisfy $1<u<v<w<14$. Then insert the gene segment between $u$ and $v$ (including $u$ and $v$ ) after $w$. 


\subsubsection{Choosing}

Using deterministic selection strategies, select $\mathrm{M}$ individuals with the smallest objective function value in the parent population and the progeny population to evolve to the next generation, so as to ensure that the superior characteristics of the parent are preserved.

\subsection{The Shortest Route Planning Based on Annealing Algorithm}

Applying the idea of simulated annealing in physics to the optimization problem can get the simulated annealing optimization method. Number 1 refers to our airport. The target points numbers are $2,3, \ldots, 13$, and finally repeat our base as number 14 (so that it is easy to calculate in the program). Distance matrix $D=\left(d_{i j}\right)_{14 \times 14} \cdot d_{i j}$ represents the distance between two points $\mathrm{i}, \mathrm{j} . \mathrm{i}, \mathrm{j}=1,2, \ldots, 14$. Here $\mathrm{D}$ is a real symmetric matrix. The problem is to find a shortest route starting from point 1 , going through all the intermediate points, and reaching point 14 . The simulated annealing algorithm is described as follows:

\subsubsection{Solution Space}

The solution space $\mathrm{S}$ can be represented as a circular permutation of all fixed starting and ending points of $1,2, \ldots, 13,14$, i.e. $S=\left\{\left(\pi_{1}, \ldots, \pi_{14}\right) \mid \pi_{1}=1,\left(\pi_{2}, \ldots, \pi_{13}\right)\right.$ is the circular permutation of $\left.\{2,3, \ldots, 13\}, \pi_{14}=14\right\}($ Qing Cheng,2009: 10-10), Each circular permutation represents a route to detect 12 target points, $\pi_{i}=j$ means the initial solution can be selected as $(1,2, \ldots, 13,14)$ at the $\mathrm{j}-1$ th detection target $\mathrm{j}$, where we use Monte Carlo method to find a better initial solution.

\subsubsection{The Objective Function}

The objective function (or cost function) is the length of route that covers all targets. We require

$$
\min f\left(\pi_{1}, \pi_{2}, \ldots, \pi_{14}\right)=\sum_{i=1}^{13} d_{\pi_{i} \pi_{i+1}}
$$

The iteration consists of the following three steps below (2.4.3 to 2.4.5).

\subsubsection{New Solutions}

Set the solution of the previous iteration to $\pi_{1} \cdots \pi_{u-1} \pi_{u} \pi_{u+1} \cdots \pi_{v-1} \pi_{v} \pi_{v+1} \cdots \pi_{w-1} \pi_{w} \pi_{w+1} \cdots \pi_{14} . \not 2$ transformation method. Draw the sequence number $\mathrm{u}, \mathrm{v}$ randomly, exchange the order between $\mathrm{u}$ and $\mathrm{v}$ into reverse. And the new route is $\pi_{1} \cdots \pi_{u-1} \pi_{v} \pi_{v-1} \cdots \pi_{u+1} \pi_{u} \pi_{v+1} \cdots \pi_{14} . \notin 3$ transformation method. Draw the sequence number $\mathrm{u}, \mathrm{v}$ and $\mathrm{w}$ randomly, after inserting the route between $\mathrm{u}$ and $\mathrm{v}$ into $\mathrm{w}$, the corresponding new route is $\pi_{1} \cdots \pi_{u-1} \pi_{v+1} \cdots \pi_{w} \pi_{u} \cdots \pi_{v} \pi_{w+1} \cdots \pi_{14}$.

\subsubsection{Cost Function Differences}

For 2 transform method, the route difference can be expressed as $\left[\Delta f=\left(d_{\pi_{u-1} \pi_{v}}+d_{\pi_{u} \pi_{v+1}}\right)-\left(d_{\pi_{u-1} \pi_{u}}+d_{\pi_{v} \pi_{v+1}}\right)\right.$

\subsubsection{Acceptance Criteria}

$$
P=\left\{\begin{array}{cc}
1 & \Delta f<0 \\
\exp (-\Delta f / T) & \Delta f \geq 0
\end{array}\right.
$$

If $\Delta f<0$, accept the new route; otherwise, accept the new path with a probability of $\exp (-\Delta f / T)$, that is, using computer to generate a uniformly distributed random number rand over $[0,1]$ interval, if rand $\leq \exp (-\Delta f / T)$, accept the new route.

\subsubsection{Cooling}

We use the selected cooling coefficient $\alpha$ for cooling, and take a new temperature $\mathrm{T}$ as $\alpha T$ (here $\mathrm{T}$ is the temperature of the previous iteration). We select $\alpha=0.999$ here.

\subsubsection{The Conditions of Ending}

Using the selected final temperature $e=10^{-30}$ to determine whether the annealing process has come to an end. If $\mathrm{T} ; \mathrm{e}$, the algorithm comes to an end, and then outputs the current state.

\subsection{Fuel Consumption Model}

Voyage through multi-tasking points consumes more fuel and needs rational planning. Adequate fuel volume should be calculated before the flying and the speed should be set so as to enhance the safety and efficiency. Constraint conditions and the objective function are important components of the fuel consumption model. Considering the specific application background of aeronautical engineering in the objective function, the model has more practical value on engineering application. (Ye Yu, 2009) Considering that the route planning based on route planning is a special case based on regional planning in mathematical models, the following models are based on regional planning. 


\subsubsection{The Basic Assumption}

(1) Great circle route assumotin The aircraft flies in a great circle route between the two points and the flight altitude remains unchanged. The aircraft routes are usually determined by the geographic coordinates, and the voyage is often farther, so the route is considered as a great circle route. Route distance $S=R \theta$. In this formula, R is the Earth's radius of curvature. (2) Ignore the impact of the weather. In a real environment, the power of the wind and the wind direction affect the air refueling route planning to a certain extent. (3) All the tankers and receivers are the same type. (4) Ignore the time consuming of the docking process . (5) The landing fuel consumption is a constant value. (6) The load of the aircraft meet the requirements. (7) The aircraft flies at the fuel-saving rate of the longest endurance to ensure the longest flight time with a certain amount of fuel. (8) The percentage of standby fuel carried by the aircraft is a constant value.

\subsubsection{Model Constructing}

According to the fuel consumption parameter curve of different types of aircraft at a certain altitude, we obtained the fuel consumption efficiency function of flight unit distance under a certain weight and altitude, which is called the fuel consumption efficiency function $F E(W, H)$. It represents the flight distance under unit fuel consumption. W represents the total weight of the aircraft, and ew is the net weight of the aircraft $W=e w+c+f, \mathrm{c}$ is the aircraft load, $\mathrm{f}$ is the weight of the aircraft's fuel. Each curve in Figure 2-5-1 shows the fuel consumption of the aircraft under different weight. For example, the points on the 1200t curve indicate the fuel consumption under different Mach. Each point in max endurance mode indicates the fuel efficiency in the max endurance mode. By connecting points in max endurance mode on each curve, we can obtain the fuel consumption efficiency under a certain cruising altitude, the max endurance condition and different weights due to the fuel consumption. Therefore, the function of fuel consumption efficiency $W=e w+c+f$ is obtained,

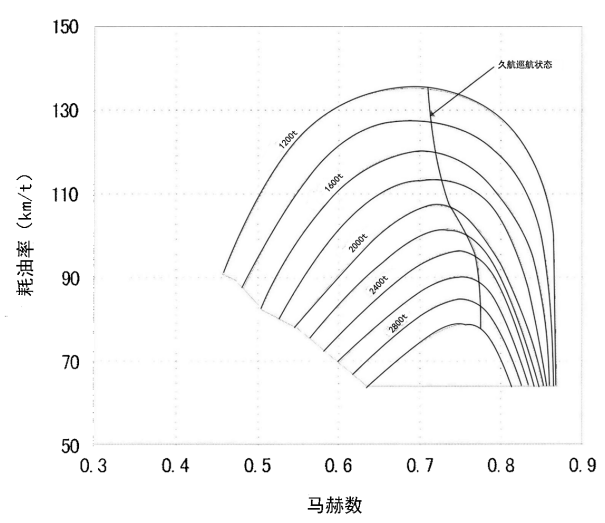

Figure 1. Fuel Consumption Parameter Curve

For the fuel consumption efficiency function $F E(W, H)$, we can obtain as a function of the flight distance at a certain initial quantity of fuel through integral: $R\left(f_{0}, c_{0}, H\right)=\int_{0}^{f_{0}} F E\left(e w+c_{0}+f\right) d f\left(f_{0}\right.$ is the initial volume of fuel). So when the aircraft fuel changes from $\mathrm{f} 0$ to $\mathrm{f}$, the flight distance is $d=\int_{f_{1}}^{f_{0}} F E\left(E W+c_{0}+f\right) d f$.

\subsection{Route Smoothing Based on Cubical Smoothing Algorithm with Five-point Approximation}

As the target points are discrete, not continuous, so yawing will appear in the simulation. Therefore, it is necessary to smooth the heading direction in order to eliminate the yawing, or the sharp turn will cause damage to the aircraft parts, increasing the costs. Cubical smoothing algorithm with five-point approximation is a commonly used smoothing algorithm based on the least-squares method. In this paper, a curve fitting and smoothing process is performed for the route and direction above. 2.6.1 The Principle of Least Square Method The basic principle of the least-squares method is to test the data with function relationship $x_{i}, y_{i}(i=0,1,2, \ldots, n)$, which can be fitted with $\mathrm{m}$-order $\left(\mathrm{m}_{i}=\mathrm{n}\right)$ polynomials.

$$
P_{m}(x)=a_{0}+a_{1} x+a_{2} x^{2}+\ldots+a_{m} x^{m}=\sum_{j=0}^{m} a_{j} x^{j}
$$

Using the least squares method to determine the coefficients $a_{j}(j=0,1,2, \ldots m)$. If we set the dispersion $x_{i}$ at the node 
$R_{i}=P_{m}\left(x_{i}\right)-y_{i}$, so fitting the least squares method is to find $y=P_{m}(x)$, and to sum up the squares of dispersion:

$$
\sigma=\sum_{i=0}^{m} R_{i}^{2}=\sum_{i=0}^{n}\left[P_{m}\left(x_{i}\right)-y_{i}\right]^{2}=\sum_{i=0}^{n}\left(a_{0}+a_{1} x_{i}+a_{2} x_{i}{ }^{2}+\ldots+a_{m} x_{i}^{m}-y_{i}\right)^{2}
$$

Take the minimum. The problem of curve fitting is $\sigma=\sigma\left(a_{0}, a_{1}, a_{2}, \ldots, a_{m}\right)$ to find the $a_{0}, a_{1}, a_{2}, \ldots, a_{m}$ that minimize the multivariate function. The following equation must be satisfied:

$$
\frac{\partial \sigma}{\partial a_{k}}=0(k=0,1,2, \ldots, m)
$$

Taking partial derivation:

$$
\begin{gathered}
\frac{\partial \sigma}{\partial a_{k}}=\sum_{i=0}^{n} 2\left[P_{m}\left(x_{i}\right)-y_{i}\right] \\
\frac{\partial P_{m}\left(x_{i}\right)}{\partial a_{k}}=2\left\{\sum_{j=0}^{m} a_{j} S_{i+k}-t_{k}\right\} \\
S_{j+k}=\sum_{i=0}^{n} x_{i}^{j+k}, t_{k}=\sum_{i=0}^{n} y_{i} x_{i}^{k}, \\
S_{0}=\sum_{i=0}^{n} x_{i}^{0}=1+1+\ldots+1=n+1 \\
(k=0,1,2, \ldots, m ; j=0,1, \ldots, m)
\end{gathered}
$$

Therefore

$$
\sum_{j=0}^{m} a_{j} S_{i+k}=t_{k},(k=0,1, \ldots, m)
$$

Then:

$$
\left\{\begin{array}{c}
S_{0} a_{0}+S_{1} a_{1}+S_{2} a_{2}+\ldots+S_{m} a_{m}=t_{0} \\
S_{1} a_{0}+S_{2} a_{1}+S_{3} a_{2}+\ldots+S_{m+1} a_{m}=t_{1} \\
\ldots \ldots \ldots \ldots \ldots \ldots \ldots \ldots \ldots \ldots \ldots \ldots \ldots \\
S_{m} a_{0}+S_{m+1} a_{1}+S_{m+2} a_{2}+\ldots+S_{2 m} a_{m}=t_{m}
\end{array}\right.
$$

The above equation is called the normal equation of least squares method. Solving the normal equations above to obtain coefficients $P_{m}(x)$ of polynomial $a_{j},(j=0,1,2, \ldots m)$, which is the method of fitting the data by least squares. It can be proved that the normal equations above have $a_{j}$ a unique solution, and the m-order polynomial $P_{m}(x)$ can indeed minimize the sum of squares $\sigma$. Therefore, we obtain $y=P_{m}(x)$, which is the fitted polynomial. 2.6.2 Cubical Smoothing Algorithm with Five-point Approximation cubical smoothing algorithm with five-point approximation is a very efficient method for smoothing data. The basic theory is to assume that the data used is a test result at an equidistant point of an unknown function $f(x)$ and that the spacing has been small enough to enable a m-order polynomials representing $f(x)$ accurately. Then, using an m-order polynomial to fit $n$ adjacent values $(m ; n)$. The outcome is substituted for the original data to eliminate the convexity and smoothing data. The smoothing method with cubical smoothing algorithm with fivepoint approximation is described below: Suppose the direction value $h_{0}, h_{1}, h_{2}, h_{3}, \ldots, h_{n}$, the smoothed direction value is $h_{0}{ }^{\prime}, h_{1}{ }^{\prime}, h_{2}{ }^{\prime}, h_{3}{ }^{\prime}, \ldots, h_{n}{ }^{\prime}$, the smoothing formula is

$$
\left\{\begin{array}{l}
h_{0}{ }^{\prime}=\frac{1}{70}\left(69 h_{0}+4 h_{1}-6 h_{2}+4 h_{3}-h_{4}\right) \\
h_{1}{ }^{\prime}=\frac{1}{35}\left(2 h_{0}+27 h_{1}+12 h_{2}-8 h_{3}+2 h_{4}\right)
\end{array}\right.
$$

The rest of the points

$$
\left\{\begin{array}{l}
h_{j}{ }^{\prime}=\frac{1}{35}\left(-3 h_{j-2 h}+12 h_{j-1}+17 h_{j}+12 h_{j+1}-3 h_{j+2}\right),(j=2,3, \ldots,(n-2)) \\
h_{n-1}{ }^{\prime}=\frac{1}{35}\left(2 h_{n-4}-8 h_{n-3}+12 h_{n-2}+27 h_{n-1}+2 h_{n}\right) \\
h_{n}{ }^{\prime}=\frac{1}{70}\left(-h_{n-4}+4 h_{n-3}-6 h_{n-2}+4 h_{n-1}+69 h_{n}\right)
\end{array}\right.
$$

\subsection{Simulation Results}

Assuming there are 12 target points need to be reconnaissance located in an area of $1000 \mathrm{~km} 1000 \mathrm{~km}$ (as 10001000 grid map, grid resolution of $1 \mathrm{~km} 1 \mathrm{~km}$ ). At the point $(1,1)$ is the starting point of the aircraft, where an airplane departs, plans its route, traverses all the target points and returns to the departure airport. The plane carry 1000t fuel while taking off, and its total weight is $2400 t$. The simulation results are as follows: 

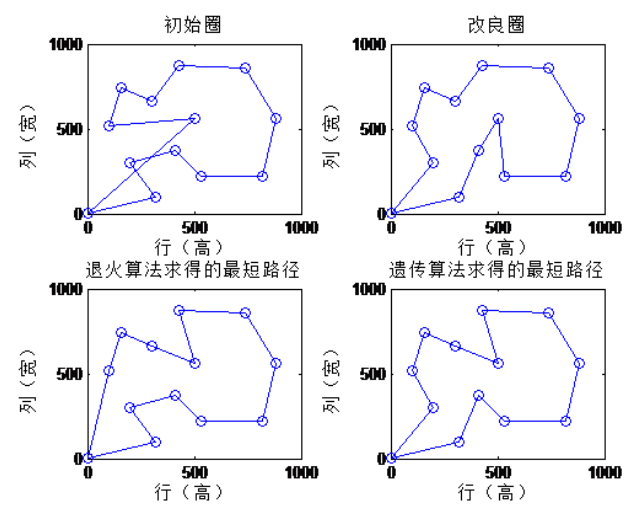

Figure 2. The Simulation Result

Model $\phi$ : initial circle We use MATLAB programming to calculate, and as a result, the length of the initial circle is 4044.8 kilometers. The route of the initial circle is 1-11-3-2-4-5-8-9-10-12-7-13-6-1. Model II: Improved Circle We use MATLAB programming to calculate, and as a result, the length of the modified circle is 3683.7 kilometers. The route of the modified circle is 1-11-2-6-4-5-8-9-10-12-7-13-3-1. Model $\phi$ : Using annealing algorithm to find the shortest route We use MATLAB programming to calculate, and as a result, the shortest route is 3716.2 kilometers. The shortest route is 1-11-3-2-4-5-8-9-10-6-12-7-13-1. Model $\phi$ : Using genetic algorithm to find the shortest route We use MATLAB programming to calculate, and as a result, the shortest route is 3618.7 kilometers. The shortest route is 1-11-2-4-5-8-910-6-12-7-13-3-1. The most accurate results are obtained by using genetic algorithms.

Table 1. Table title (Shortest Route Length Obtained by Four Models)

\begin{tabular}{lcccc}
\hline model & initial circle & Improved Circle & annealing & Genetic \\
\hline Route length $/ \mathrm{km}$ & 4044.8 & 3683.7 & 3716.2 & 3716.2 \\
\hline
\end{tabular}

According to the aircraft engineering parameters, we can get the fuel consumption efficiency of the aircraft:

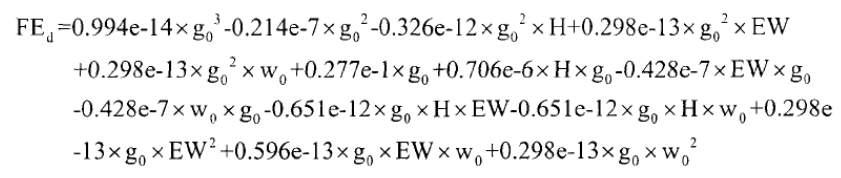

EW is the net weight of the aircraft, $g_{0}$ is the load of the aircraft, $w_{0}$ is the fuel weight of the aircraft, and $\mathrm{H}$ is the flight altitude. Iterating the aircraft fuel consumption to obtain the optimal route planning based on genetic algorithm.

Table 2. Table title ( Data of the Route Planning by Genetic Algorithm)

\begin{tabular}{lccc}
\hline Route / km & Time consuming / $\mathrm{h}$ & Fuel consumption / & Average speed $(\mathrm{km} / \mathrm{h})$ \\
\hline 3618.7 & 4.1 & 485.9 & 893.5 \\
\hline
\end{tabular}

Finally, in order to reduce the cost caused by the sharp turns of the aircraft, cubical smoothing algorithm with five-point approximation of the route is carried out. The results is as follows:

\subsection{Model Testing}

In order to verify the stability of the model, we make some slight changes on the target point. The model of the improved circle, the annealing operation model and the genetic algorithm model were recalculated. From the test results, it can be concluded that the stabilities of the three models are all good, in which the genetic algorithm has the highest accuracy and the smallest difference with the original one. So the genetic algorithm is regarded as the optimal model. 


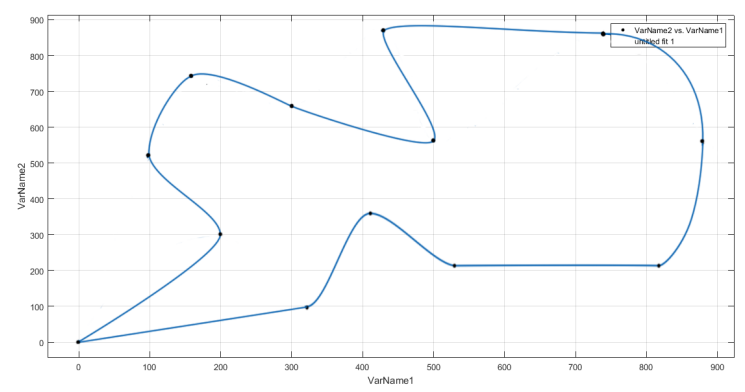

Figure 3. The Route After Smoothing

\section{Conclusion}

Genetic algorithm can be widely applied to all kinds of problems, its main feature is that it operates directly with the structure object. There is no limitation of derivation and function continuity; with inherent implicit parallelism and better global optimization ability; using probabilistic optimizing method can automatically obtain and guide the optimization of search space, and can adaptively adjust the search direction, which do not need to determine the rules. According to the requirements of route planning, this paper proposes a route planning method based on genetic algorithm. Simulation results show that the genetic algorithm has a good convergence, less computational time and high robustness when calculating the precision requirements. Based on the non-restricted airplane route planning problem, adding some the restriction conditions and expanding the algorithm, it can be applied to a wider range of restricted route planning problems.

\section{References}

WANG Shu-Xi. (2014). Multiple Adjacent Points and Multiple Shortest Routes Problem in Dijkstra Algorithm. Computer Science., 41(6), 217-224. http://www.cqvip.com/QK/92817X/201205/

Shui Wei, Ge Yan, Han Yu, et al. (2011). UAV route planning based on hybrid ant colony algorithm. Journal of System Simulation, 23(3), 574-576. http://www.cqvip.com/QK/96569X/201103/

Kong, Yiyi. (2013). Usng Chaos Genetic Algorithm to Find the Optimal Constrained Traveling Salesman Route. . Microelectronics and Computer, 30(8), 75-78. http://www.cqvip.com/QK/92040X/201308/

Hua Yu. (2009). Airplane Air Refueling Route Planning Based on Genetic Algorithm. Fudan University. https://doi.org/10.7666/d.y1968230

Jiaxin Yao, Chunlai Zhou, \& Dongping Liu. UAV Route Planning and Simulation with Improved Genetic Algorithm. Computer Simulation, 30(12), 17-20. http://www.cqvip.com/QK/92897X/201312/

Shuyuan Zhang, \& Cong Guo. (2009). The Shortest Path Problem Based on Genetic Algorithm and its Matlab Realization, (12), 104-105. Transpoworld. http://www.cqvip.com/QK/98465X/200912/

Bo He, Lijuan Qin, \& Mingyang Jiang. (2010). Research on the Path Planning Methods for Mobile Robots Based on an Improved Genetic Algorithm. Computer Engineering and Science, 32(7), 104-107. http://www.airitilibrary.com/Publication/alDetailedMesh?docid=P20091029004-201007 -201007080027-201007080027-104-107

Qing Dai, (2009). Path Planning for Robots based on Annealing Algorithm 10 : 10-10. Science and Technology Information http://www.cqvip.com/QK/87241X/200910/

\section{Copyrights}

Copyright for this article is retained by the author(s), with first publication rights granted to the journal.

This is an open-access article distributed under the terms and conditions of the Creative Commons Attribution license (http://creativecommons.org/licenses/by/4.0/). 